\title{
When to Operate on Open Angle Glaucoma
}

\author{
P. G. WATSON \\ Cambridge
}

\begin{abstract}
Summary
The aim of treatment in primary angle glaucoma should be to reduce the intraocular pressure throughout the 24 hours to a level where no damage is done to the optic nerve head; thus preventing any field defect. Open angle glaucoma as it is presently defined requires a significantly raised intraocular pressure to be associated with detectable changes in the optic nerve and a consequent visual field defect. The goal is therefore unattainable whilst this definition remains. The best that can be achieved is to reduce the intraocular pressure to such a level that no further disc damage occurs. Unfortunately, because there is no method of measuring the intraocular pressure over 24 hours, large diurnal rises of pressure may well remain undetected, producing irreparable damage to the disc. Furthermore, significant nerve fibre loss occurs before any field defect is detected even by the most sophisticated testing techniques and some patients develop field defects in spite of normal intraocular pressures. Treatment therefore is largely empirical, relying on the coarse assessment of visual fields, intermittent measurements of intraocular pressure and the clinical observation of the optic nerve head.

Chronic open angle glaucoma is a disastrous, insidious disease destructive to the optic nerve head. It is difficult to diagnose before serious damage has occurred and it is difficult to monitor once it has been discovered. Medical therapy was successful in preventing field loss in one third of patients when the medication was given infrequently and in low dosage. Higher dosages were poorly tolerated and the drugs were often not taken by the patient. No advantage was obtained by increasing the strength and frequency of the medication because any improvement was transient.

In our population, trabeculectomy was effective in reducing the intraocular pressure to normal limits throughout the 24 hours and the complication rate was low enough to justify operating on all those who have not responded to low dose medical therapy (Timoptol 0.25 per cent and Pilocarpine 2 per cent tid) and or laser trabeculoplasty before further field loss occurred. The surgical results are presented.
\end{abstract}

\section{Selection of Patients for Medical Treatment}

Whilst it is obviously desirable to treat a patient with glaucoma as early as possible in order to prevent progression of the disease, it is so far impossible to do so until significant changes have taken place. Certain risk factors are, however, known to be associated with the loss of visual field.

\section{Risk Factors for Field Loss in Glaucoma}

Persistently raised pressure beyond $30 \mathrm{mmHg}$

Rising intraocular pressure with time especially in elderly patients
Glaucoma in the fellow eye

Raised pressure in high myopia

Optic disc haemorrhage

require to be treated medically.

Those with:

Vertical enlargement of the optic cap

Peripapillary atrophy

A family history of glaucoma

Retinal and systemic vascular disease

Pigment dispersion

Pseudo exfoliation

or those using systemic steroids need to be 


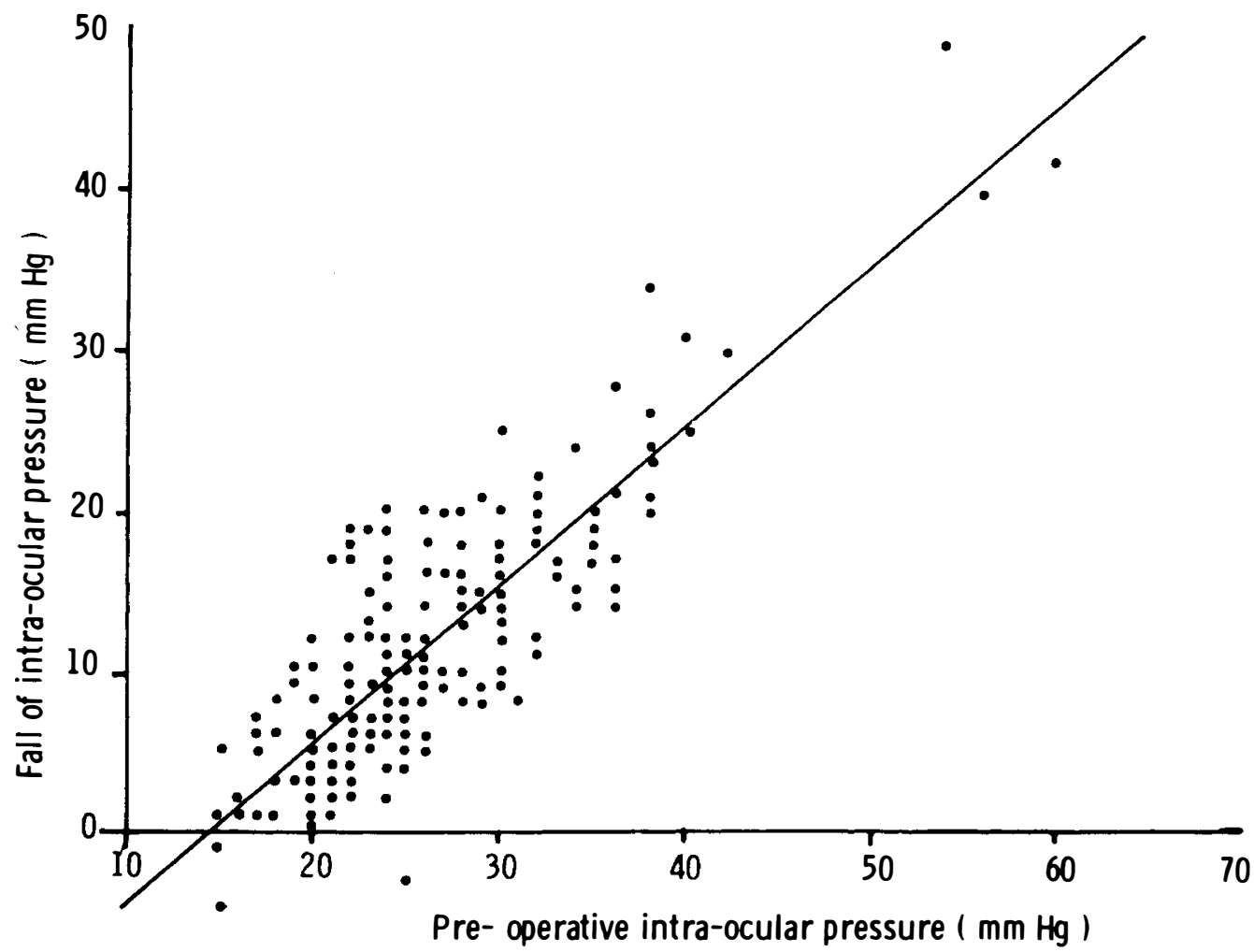

Fig. 1. Regression analysis of patient (258 eyes) operated on for chronic open-angle glaucoma, using a limbusbased flap for trabeculectomy.

observed at frequent intervals bearing in mind that all medication has serious side-effects. These patients should be treated if their intraocular pressure rises.

If a patient has already developed marked disc and visual field changes which are not purely ischaemic he should be treated.

\section{Selection of Patients for Further Treatment}

(1) Progression of visual field defects in spite of medical treatment

(2) Development of side effects from the medication

(3) Failure to control the intraocular pressure throughout the 24 hours

(4) Progressive increase in strength or frequency of medication required

A patient who develops progressive field change in spite of medical therapy is a candidate for laser or surgical treatment whatever the reason for the progression of the disease, be it non-compliance with the treatment or further physical changes in the eye.
Treatment, once started, brings with it a series of problems and complications. It is rare indeed for there not to be some side effect of glaucoma therapy of whatever type. The best tolerated of medical therapy is with beta blockers, but cardiac side effects and asthma are not uncommon. The miotics, because of their effect on the pupil, cause at best dimming of the image and at the worst variable and intolerable myopia. Systemic absorption occasionally also causes parasympathetic stimulation. Prolonged use of the adrenergic drugs gives rise to redness and discomfort of the eyes which many patients find intolerable. Acetazolamide given over a prolonged period produces side effects; tingling of the fingers, potassium depletion, diarrhoea and malaise in almost every patient, to such an extent that it should only be used as a continuous therapy in the most extreme circumstances. If there is an insistence that all patients should have had 'maximum tolerated medical therapy' before anything further is done, many, even if closely supervised, will have lost a great deal of field before any decision is reached as to their further care. Increasing strength and frequencies of 


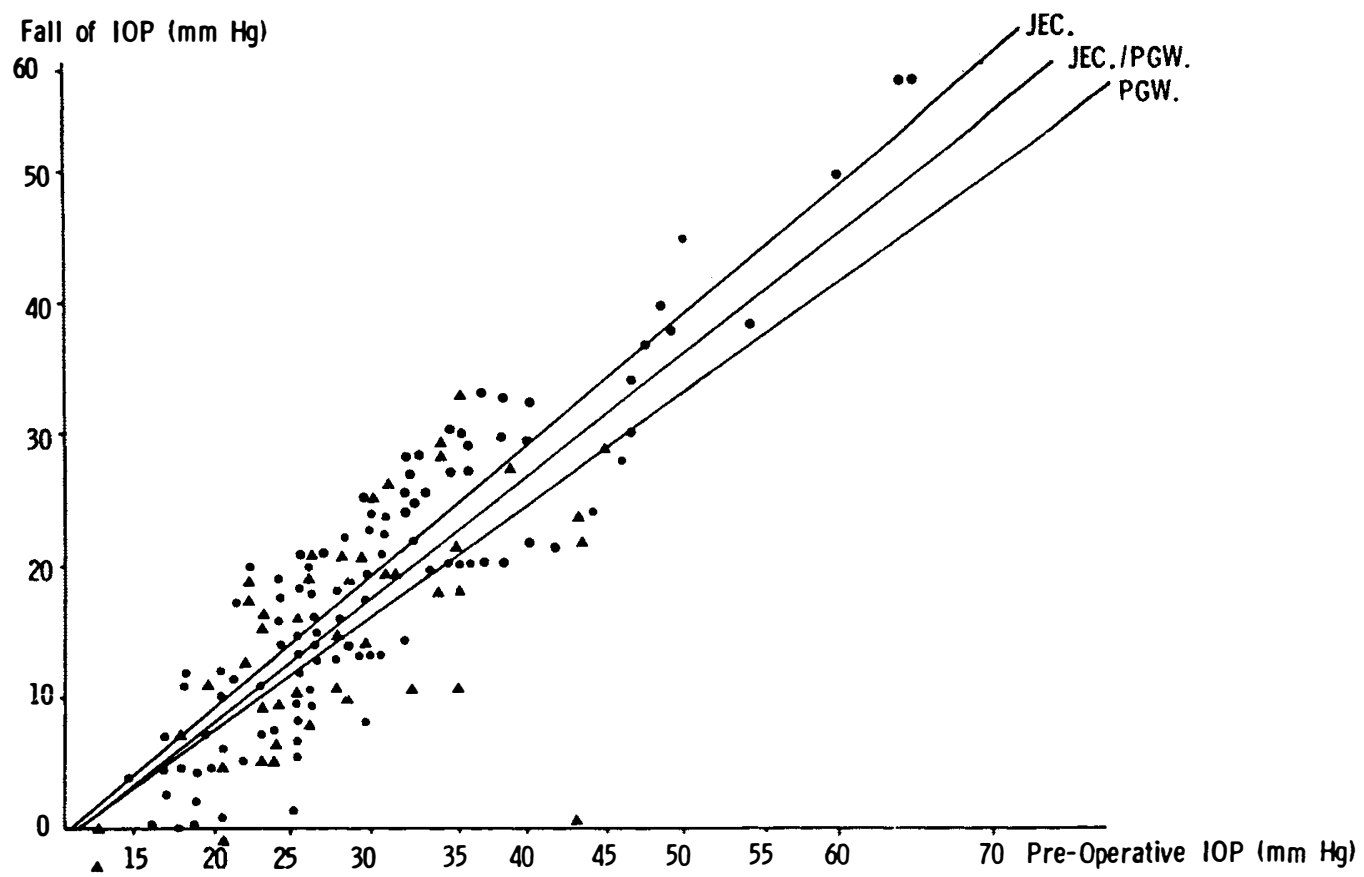

Fig. 2. Regression analysis of results of trabeculectomy using a fornix-based flap, carried out in 253 eyes with chronic open-angle glaucoma. The meshwork was excised anterior to the scleral spur (JEC) ( $\mathbf{\Delta})$ and posterior to the spur (PGW) (O). No difference was observed. (IOP = intraocular pressure.) (A single symbol may represent more than one point.)

treatment are often tried in order to bring the intraocular pressure under control during the 24 hours. However, such a technique is not likely to be successful because it has been shown that whilst parasympathomimetic agents in low dosage open the trabecular spaces and increase the facility of outflow, higher or prolonged dosage leads to cellular loss and fibrotic changes in the meshwork. Treatment should therefore be started with Timoptol 0.25 per cent bd and continued if the intraocular pressure is reduced to below $20 \mathrm{mmHg}$ or to the level required to prevent field loss, whichever is the lower. If further medication is considered necessary then Pilocarpine up to, 2 per cent three times a day is added. Over 5 years 36 per cent of our patients have been controlled on this regime and have tolerated therapy well. The rest have required alternative treatment.

\section{Selection of the Type of Alternative Treatment}

If medical treatment for whatever reason has been unsuccessful there are two possible alternatives for further treatment; laser trabeculoplasty and trabeculectomy. The choice will depend on many factors, not all of them medical. Laser trabeculoplasty is not an easy technique. It is time consuming and requires a suitable laser and a skilled operator with sufficient time to perform the procedure carefully and well. Patients with narrow angles (which constitutes the majority in some populations) cannot be lasered satisfactorily and most of the patients will require to continue on their preoperative medications after trabeculoplasty. However, the procedure is an out-patient one and suitable for many who for various reasons are unsuitable for admission and surgery.

Trabeculectomy is the surgical treatment of choice in chronic open and closed angle glaucomas. It is a predictable procedure as far as its effect on intraocular pressure is concerned (Fig. 1). Several different methods of providing a controlled fistulisation are used but all work by allowing the aqueous to by-pass the trabecular meshwork and flow into the subconjunctival space and approximating the intraocular pressure to that of the episcleral venous pressure. The precise technique of achieving this makes no difference to the effectiveness of the procedure (Fig. 2). The regression analysis of the results shows that whatever the initial intraocular pressure the final intraocular pressure will be within the normal range (the higher 
Table 1. Comparative results of different techniques of trabeculectomy (follow-up greater than one year)

\begin{tabular}{|c|c|c|c|c|}
\hline \multirow[b]{2}{*}{ Variables } & \multicolumn{2}{|c|}{ Limbus-based flap } & \multicolumn{2}{|c|}{ Fornix-based flap } \\
\hline & $\begin{array}{l}\text { Anterior } \\
\text { Approach }^{\mathrm{a}}\end{array}$ & $\begin{array}{l}\text { Posterior } \\
\text { Approach }^{\mathrm{b}}\end{array}$ & $\begin{array}{l}\text { Anterior } \\
\text { Approach }^{\mathrm{a}}\end{array}$ & $\begin{array}{l}\text { Posterior } \\
\text { Approach }^{b}\end{array}$ \\
\hline Total eyes & 115 & 101 & 140 & 96 \\
\hline Control with IOP without medication & $82 \%$ & $81 \%$ & $85 \%$ & $82 \%$ \\
\hline \multicolumn{5}{|l|}{ Visual acuity: } \\
\hline Better & $4.3 \%$ & $4.9 \%$ & $7.1 \%$ & $16.6 \%$ \\
\hline Unchanged & $87.6 \%$ & $90 \%$ & $87.1 \%$ & $69.8 \%$ \\
\hline Worse & $8.7 \%$ & $4.9 \%$ & $5.7 \%$ & $13.5 \%$ \\
\hline Cystic blebs & $36 \%$ & $33 \%$ & $26 \%$ & $16 \%$ \\
\hline
\end{tabular}

a In the anterior approach (Cairs [2a]), the cornea is first cut parallel to the trabecular meshwork and is then excised from before, backward to, but not including, the scleral spur.

${ }^{b}$ In the posterior approach (Watson [10a]), the first incision is behind the scleral spur which is dissected off the underlying ciliary muscle. The trabecular tissue is then removed from behind, forward.

Table 2. Complications following trabeculectomy

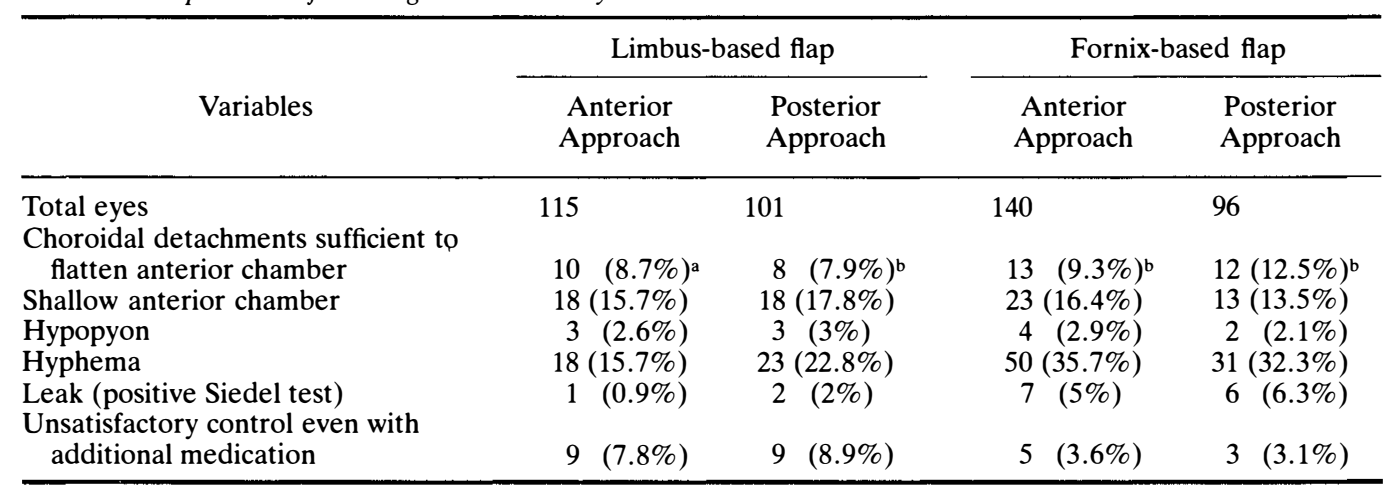

a 3 drained.

b 2 drained.

the pressure the greater the fall). Some discussion has arisen as to whether a fornix based conjunctival flap or a limbal based conjunctival flap is the most efficient. Table I shows there is no difference in the control of intraocular pressure but less cystic blebs occurred when a fornix based flap was used.

With the certain knowledge that in over 80 per cent of patients the intraocular pressure can be kept below $20 \mathrm{mms} \mathrm{Hg}$ throughout the 24 hours, ${ }^{1}$ the possibility of developing complications is the only thing to prevent all patients who have proven glaucoma being offered surgery as soon as the diagnosis is made. This has to be the individual judgement of each surgeon. Table II shows the immediate post-operative complication rate. Although between a quarter and a third of the patients had some minor complication, the major complication rate was less than 0.5 per cent. The only unquantifiable long term complication is that of the maturation of cataract in those who already have some lens opacity. Our investigations into this aspect are still incomplete but those indicate that in . eyes without significant lens change pre-operatively there is no change over 5 years but cataracts have matured more rapidly in the operated than in the fellow unoperated eyes if there was significant cataract at the time of surgery.

\section{References}

'Watson, PG and Grierson, I: Place of Trabeculectomy in the Treatment of Glaucoma. Ophthalmology 1981; 88, 175-96. 